Mloman 2016, 34(1), 93-102

Revista de Psicologia, Ciències de l’Educació i de l'Esport

ISSN: $1138-3194$

Copyright @ 2016

www.revistaaloma.net

\title{
Propuesta didáctica en la enseñanza del waterpolo
}

Yurema Sabio-Lago, Josep Solà-Santesmases \& Myriam Guerra-Balic

Universitat Ramon Llull

Recibido: 9-9-2015

Aceptado: 27-2-2016

\section{Propuesta didáctica en la enseñanza del waterpolo}

Resumen. El waterpolo requiere una serie de fundamentos técnicos, tácticos, de cualidades psicosociales y de preparación física que los jugadores y las jugadoras irán asumiendo de manera progresiva con la edad.La metodología de aprendizaje es muy importante a la hora de enseñar cualquier deporte. Además, el waterpolo se practica en el medio acuático, que no es el medio natural del ser humano, lo cual le da una especial singularidad respecto al resto de deportes colectivos. En nuestra disciplina, las referencias bibliográficas relativas a sobre cómo llevar a cabo este proceso son limitadas y creemos que hay que plantearse, además de qué enseñar, cómo y cuándo enseñarlo. Nuestro objetivo es desarrollar una propuesta de los planteamientos pedagógicos que hay que seguir en los diferentes estadios de enseñanza del waterpolo teniendo en cuenta los objetivos y contenidos que deberán ser asumidos en cada una de las categorías.

Palabras clave: deporte; waterpolo; planteamiento pedagógico; estilos de enseñanza

\section{Educational program for the teaching of water polo}

Summary. Water polo requires a series of technical and tactical foundations and a framework of psycho-social qualities and physical fitness, with players gradually acquiring these attributes as they get older.

Learning methods are critical to the teaching of sports. In addition, water polo is played in water, which is not the natural environment for human beings, a fact that makes it unique among team sports. In our discipline, there are few published references on how to carry out this process, and we believe that it is necessary to pose questions not only of what to teach, but of when and how to teach it.

Our objective is to develop a program that details the educational approach to be followed at the various stages of the teaching of water polo, one that bears in mind the objectives and contents that must be learned in each category.

Keywords: sports; water polo; educational approach; teaching styles 


\section{Introducción}

El waterpolo es un deporte colectivo que se practica en el medio acuático y que tiene su origen en Inglaterra hacia 1869. Está sujeto, normativizado e institucionalizado, y lo practican dos conjuntos de siete jugadores o jugadoras por campo (seis y el portero o la portera) en una superficie limitada de piscina. La finalidad es introducir la pelota en la portería contraria (Lloret, 1994).

El waterpolo se clasifica como deporte sociomotriz de cooperación/oposición, donde son imprescindibles el factor de decisión y la comunicación. Es uno de los deportes de mayor complejidad en cuanto a su estructura, según Parlebas (1981). Como en todos los deportes colectivos (baloncesto, balonmano...), en el waterpolo tanto el reglamento (por ejemplo, pasar de los 7 $\mathrm{m}$ a $5 \mathrm{~m}$ para el lanzamiento directo, pasar de poder levantar dos brazos a solo poder levantar uno como jugador o jugadora de campo), como en los aspectos técnicos, tácticos y condicionales han ido evolucionando. El waterpolo actual es dinámico y se exige mucha fuerza explosiva y resistencia, a la vez que un somatotipo específicamente idóneo (Vila, Ferragut, Abraldes, Rodríguez \& Argudo, 2010).

Cancela y Lago (2001) proponen una enseñanza integral de todos los elementos que componen el waterpolo secuenciando los contenidos técnico-tácticos en seis fases: 1. Disponibilidad acuática, 2. Construcción de la relación con el móvil, 3. Construcción de la acción de juego con presencia de adversario, 4. Construcción de la acción de juego con la presencia de compañero, 5. Construcción de la acción de juego con presencia de adversario y compañero y 6. Desarrollo de la adecuación espacio-temporal. Para cada fase se deben presentar, progresivamente, unos objetivos y unos principios ofensivos y defensivos tanto individuales como colectivos, y unas situaciones específicas que hay que desarrollar en cada etapa.

La revisión bibliográfica aporta algunas propuestas. Así, López (1995) propone una estructuración de cuatro niveles de desarrollo en la iniciación al waterpolo: Nivel I, de 8 a 11 años, que valora como experimentación con el medio; Nivel II, de 11 a 12 años, donde se pasa de aspectos globales a la introducción y conocimiento más en profundidad del waterpolo; Nivel III, de 13 a 14 años, que marca la especialización deportiva; y Nivel IV, de 15 a 16 años, señala el final de la especialización deportiva. Por otro lado, Argudo y García (1996) plantean el aprendizaje de los sistemas tácticos de juego libre, de organización simple o codificados en cada marco situacional. En cambio Feu (1999) plantea reducir la incertidumbre del juego disminuyendo el número de estímulos que hay que atender y jerarquizarlos dando prioridad a los más importantes para conseguir los objetivos deportivos. García (1999) sugiere un manual para la enseñanza del waterpolo y García (2000) presenta una programación anual para un grupo de edad integrando acondicionamiento general, acondicionamiento específico, habilidades técnicas y juego o formas jugadas. Por último, Fernández (2004) propone una unidad didáctica de ocho sesiones para niños y niñas de 11 años.

En esta primera relación de autores se echa de menos un planteamiento pedagógico organizado del waterpolo, lo que justifica el esfuerzo de proponer una metodología de aprendizaje para dicho deporte. Una notable referencia en este aspecto nos la ofrece Blázquez (1995), quien determina los aspectos que influyen en la enseñanza deportiva, y por lo tanto del waterpolo, y estos son los siguientes:

a) Las características del individuo que aprende/entrena, incluyendo los siguientes factores: las capacidades físicas básicas, la etapa de crecimiento y maduración, la personalidad y el comportamiento, la motivación, las cualidades motoras previas y la experiencia anterior, la capacidad de aprendizaje, el estado de salud física, psíquica y social, las condiciones socioeconómicas y el ambiente cultural donde vive, así como otros factores que puedan incidir en su personalidad y comportamiento.

b) Las características de la actividad deportiva y de su estructura lógica. Todo lo que envuelve al waterpolo desde un aspecto formal y funcional, como son las reglas, los principios, la lógica interna del juego, entre otras.

c) Los objetivos que se pretenden conseguir, como mejorar sus habilidades motrices, perfeccionar y progresar en la ejecución, estar dispuesto a rendir de manera óptima, favorecer el aspecto social (cooperación e integración), llegar a ser autónomo, adaptarse al deporte y especializarse en este.

d) Los planteamientos pedagógicos o métodos didácticos, los cuales, según Delgado (1993), tratan de situar a través de la acción docente los nuevos aprendizajes al alcance de los jugadores y las jugadoras.

Los objetivos específicos del waterpolo deben concretarse para cada etapa según la edad y/o el nivel de los jugadores y las jugadoras. Dividiremos las etapas del waterpolo de la misma manera que en la Federació Catalana de Natació, en la sección de waterpolo, en iniciación, desarrollo, perfeccionamiento y rendimiento.

En la etapa de INICIACIÓN (benjamín y alevín, de 9 a 12 años) se deberán adquirir las competencias de dominar el medio acuático, la técnica individual y el espacio táctico. Lloret y Baella (1996) afirman que los objetivos ofensivos de la iniciación son desmarcarse, conservar el balón y lanzar a gol desde posiciones cercanas a portería; mientras que los objetivos defensivos son individualizar el marcaje, recuperar el balón y anticiparse al adversario.

Por otro lado, Ortega (1996) realiza una propuesta para la iniciación al waterpolo indicando las características especiales y los aspectos metodológicos y de contenido de la iniciación que divide en Nivel I (6-9 años) y Nivel II (10-11 años). En la iniciación marca como contenidos las habilidades básicas, los juegos no reglados y la metodología global en espacios amplios, 
entre otros. Otro autor (Genoves, 1992) señala unos objetivos de entrenamiento que los chicos de entre 10 y 13 años tienen que desarrollar, que en resumen son proporcionar las cualidades básicas para que en un futuro puedan desempeñar más ampliamente un alto rendimiento.

En la etapa de DESARROLLO (infantil y cadete, de 13 a 16 años) se deberá desarrollar la fuerza (de lanzamiento, de salto, de lucha y de desplazamiento) como cualidad física básica para llegar a jugar en la alta competición -sin olvidar las otras capacidades condicionales-, dominar y ampliar el juego individual (a nivel técnico) y dominar las intenciones tácticas individuales, al mismo tiempo que será preciso entrenar y dominar el juego colectivo (técnica y táctica colectiva) y los sistemas de juego. Lloret y Baella (1996) consideran la etapa de perfeccionamiento hasta los 14 años de edad, la cual coincidiría con esta, y definen los objetivos ofensivos de desmarcarse para ocupar o crear espacio, conservar la posesión del balón, conseguir gol, conocer y ocupar de forma polivalente las posiciones del sistema ofensivo básico e iniciarse en el sistema básico de superioridad numérica; y los objetivos defensivos de presionar, anticiparse/interceptar, evitar el lanzamiento a portería, conocer y ocupar de forma polivalente las posiciones del sistema defensivo básico e iniciarse al sistema básico de inferioridad numérica.

En la etapa de PERFECCIONAMIENTO (juvenil, 17-18 años) se ampliarán los objetivos de la etapa anterior, trabajándolos más a fondo. Por último, el propósito de la etapa de RENDIMENTO será intentar conseguir el máximo nivel de todas las capacidades y cualidades anteriores para llegar a ser un deportista de alto rendimiento.

En este artículo nos centraremos, sobre todo, en la etapa de iniciación y desarrollo, ya que son las etapas en las que tendremos que crear la base para poder desarrollar el juego en edades posteriores. Estamos de acuerdo con Argudo (1996) cuando afirma que los niveles de enseñanza deben confeccionarse utilizando los principios pedagógicos y técnicos. En cuanto a los planteamientos pedagógicos, cabe señalar que el entrenador o entrenadora escogerá un estilo de enseñanza u otro en función de su propia manera de pensar y actuar o del nivel del grupo con el que trabaja, entre otras características.
2. Técnicas de enseñanza, estrategias en la práctica y modelos de enseñanza de los juegos deportivos

Existen diferentes clasificaciones de estilos de enseñanza según los autores consultados, que utilizan diferentes nomenclaturas para designar un mismo término (Herbert, 1967; Stones, 1973; Delgado, 1991a; Delgado, 1991b) y que han ido cambiando con el paso de los años (Sicilia, 2001). Dependiendo de la personalidad del entrenador, del grupo, de los objetivos, de los contenidos y de las actividades de enseñanza-aprendizaje escogeremos unos u otros con tal de mejorar la relación didáctica.

Mosston y Ashworth (1993) dividen los estilos en dos grupos basados en la instrucción directa, como los estilos A-E (A: Comando Directo, B: Estilo de la práctica, C: Estilo recíproco, D: Estilo de autoevaluación y E: Estilo de inclusión) y estilos basados en la indagación (o estilos F-J), donde, por un lado, están los F-G (F: Estilo de descubrimiento guiado, G: Estilo de resolución de problemas) y, por otro lado, los H-J, estilos basados en el diseño (H: Programa individualizado, I: Estilo para alumnos iniciados, J: Estilo de autoenseñanza) (Ver Figura 1). Según Sicilia y Delgado (2002), clasificar de esta manera es un error, ya que estarían agrupando los estilos de enseñanza en función de una parte del estilo, la denominada técnica de enseñanza. La tradicional clasificación de Mosston y Ashworth (1993) no tiene en cuenta la estrategia de la práctica que plantea Delgado (1993), que tan solo aparecería de manera intrínseca en algunos estilos (en el A y B, por ejemplo, se utilizaría la estrategia de carácter analítico), pero en otros estilos podríamos utilizar las combinaciones de estrategia de la práctica que creyéramos necesarias.

Por este motivo, la clasificación que más se adecúa a nuestras necesidades es la de Delgado (1993), ya que desmenuza minuciosamente la intervención didáctica y no da pie a confusiones. Algunos estilos de enseñanza llevarán implícitas la técnica de enseñanza y la estrategia de práctica que se utilizará. Por ejemplo, en los estilos tradicionales, van implícitas la técnica de enseñanza mediante la instrucción directa y la estrategia de práctica de carácter más analítico (Ver Figura 2).

\subsection{Estilo de Enseñanza}

Delgado (1993) señala que el estilo de enseñanza (EE) es una forma peculiar de interaccionar con los alumnos/

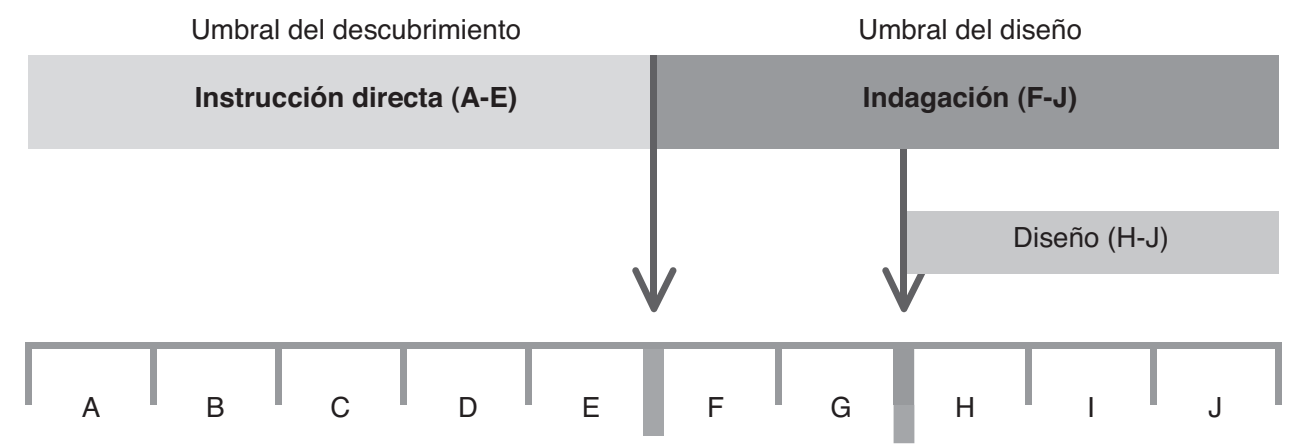

Figura 1. Espectro de los estilos de enseñanza, adaptado de Mosston y Ashworth (1986, pág. 265). 


\begin{tabular}{|c|c|c|c|c|}
\hline $\begin{array}{l}\text { FAMILIAS DE ESTILOS } \\
\text { DE ENSEÑANZA }\end{array}$ & $\begin{array}{l}\text { EE PROPUESTOS POR } \\
\text { DELGADO (1991) }\end{array}$ & \multicolumn{3}{|c|}{ EE PROPUESTOS POR MOSSTON Y ASHWORTH (1986) } \\
\hline Estilos tradicionales & $\begin{array}{l}\text { Comando directo } \\
\text { Modificación del comando } \\
\text { directo } \\
\text { Asignación de tareas }\end{array}$ & ${ }^{A}{ }^{A} B$ & & \\
\hline $\begin{array}{l}\text { Estilos que fomentan la } \\
\text { individualización }\end{array}$ & $\begin{array}{l}\text { Individualización de grupos } \\
\text { Enseñanza modular } \\
\text { Programas individuales } \\
\text { Enseñanza programada }\end{array}$ & D & 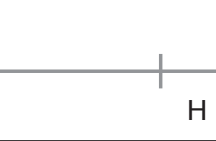 & I J \\
\hline $\begin{array}{l}\text { Estilos que posibilitan la } \\
\text { participación }\end{array}$ & $\begin{array}{l}\text { Enseñanza recíproca } \\
\text { Grupos reducidos } \\
\text { Micro-enseñanza }\end{array}$ & C & & \\
\hline $\begin{array}{l}\text { Estilos que promueven } \\
\text { la creatividad }\end{array}$ & Estilo creativo & & & \\
\hline
\end{tabular}

Figura 2. Clasificación de Mosston y Ashworth (1986) basada en la clasificación de Delgado (1991). Adaptación de Sicilia, (2001).

jugadores y que se manifiesta en las decisiones preactivas (planificación), durante las decisiones interactivas (implementación de la sesión) y en las decisiones post-activas (evaluación).

El mismo autor propone la siguiente clasificación, a la que añadiremos ejemplos prácticos aplicados al waterpolo:

\subsubsection{EE tradicionales}

El comando directo no se utiliza mucho en waterpolo, no necesitamos la simultaneidad de los movimientos ni gestos. Si se realiza un movimiento defensivo frontal, no tendrán que hacerlo todos los jugadores o las jugadoras de manera simultánea, por eso se utilizará el comando directo modificado.

El comando directo modificado se usa, por ejemplo, al realizar un movimiento defensivo frontal, dando todas las indicaciones técnicas necesarias y efectuando las correcciones pertinentes de manera colectiva e individual (Ver Figura 3).

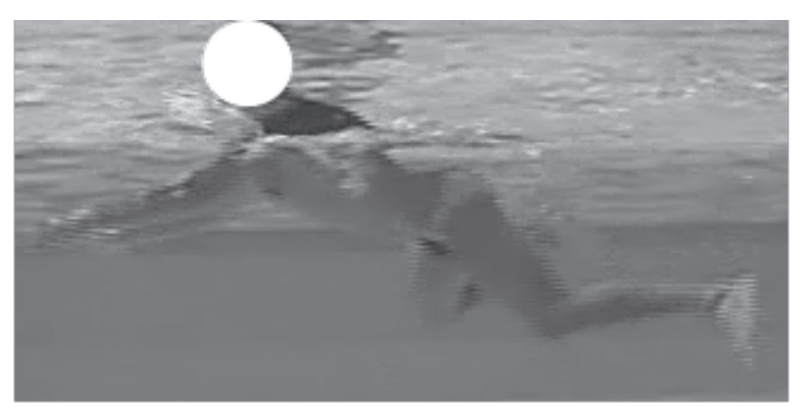

Figura 3. Desplazamiento frontal defensivo (comando directo modificado). (Imagen cedida por la Federació Catalana de Natació, contenidos del nivel I). El entrenador o entrenadora deberá indicar constantemente los ítems, de manera general y también de manera individual, puesto que no todos los jugadores y jugadoras realizarán estos ítems por igual. En posición horizontal, con la cabeza hacia abajo sin que la barbilla toque el agua, con la cadera arriba, las rodillas abiertas, dando patada de waterpolo, y la remada con las manos un poco más abiertas que a la altura de los hombros.
La asignación de tareas se usaría, por ejemplo, en el siguiente ejercicio: «ve hacia el otro lado de la piscina mediante desplazamientos defensivos». El jugador o la jugadora pueden escoger si ir de manera frontal, lateral, hacia atrás, con patada de bici u otro modo.

\subsubsection{EE que fomentan la individualización}

El trabajo en grupos de nivel, intereses, etc. se utilizaría, por ejemplo, al dividir el equipo en porteros, jugadores de arco, jugadores de boya y defensores de boya, con la finalidad de trabajar específicamente con cada grupo (Ver Figura 4).

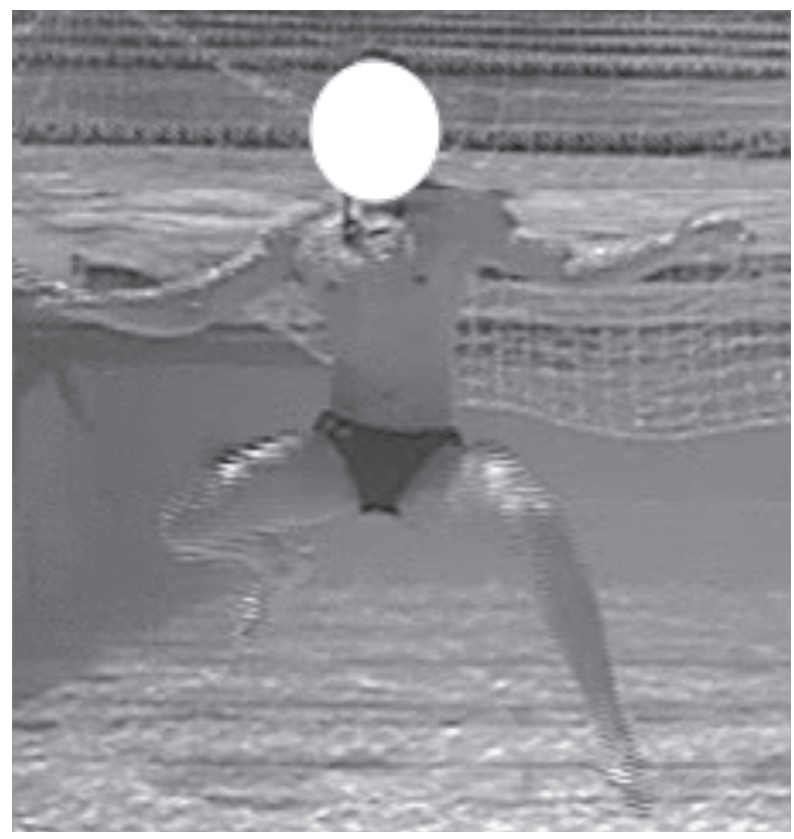

Figura 4. Desplazamiento lateral de porteros (grupos de interés). (Imagen cedida por la Federació Catalana de Natació, contenidos del nivel I). Trabajaremos solo con los porteros, en este caso, el desplazamiento lateral. Deberemos incidir igualmente en los ítems. Rodillas altas y hacia adelante, espalda recta, más remada si vamos hacia la derecha con el brazo izquierdo y a la inversa. 
La enseñanza modular se utilizaría de la misma manera que el estilo anterior, pero con grupos aún más reducidos (por nivel). Por ejemplo, el grupo de porteros lo subdividiríamos en un grupo de mayor nivel (técnico) y un grupo de menor nivel (técnico).

Los programas individuales se ven reflejados cuando, por ejemplo, el propio equipo escoge (con el respaldo del staff) el calentamiento previo del partido que van a jugar.

La enseñanza programada presenta los contenidos que hay que trabajar de manera gradual y organizada, para que los jugadores y las jugadoras participen activamente de la enseñanza. Los jugadores y las jugadoras avanzarán cuando la respuesta sea correcta. Por ejemplo, si pedimos a un jugador o jugadora que defienda en línea de pase, y lo hace correctamente, le pediremos lo mismo, y si hay falta, que ayude al defensor de boya; pero si no lo hace correctamente seguirá defendiendo en línea de pase hasta que le salga bien. De esta manera cada jugador o jugadora sigue su ritmo de aprendizaje.

\subsubsection{EE que posibilitan la participación del alumnado en la enseñanza}

La enseñanza recíproca es de fácil y útil aplicación. Los jugadores o jugadoras, colocados normalmente por parejas, conocen la tarea que hay que realizar. Mientras uno de ellos la ejecuta, el otro observa, y valora la tarea de su compañero. Es importante que el entrenador o entrenadora explique qué aspectos de la ejecución del compañero son los que tiene que observar. Por ejemplo: en la ejecución del lanzamiento, el observador tiene que fijarse en el brazo de apoyo, las rodillas, el codo...

Los grupos reducidos son varios jugadores agrupados según diferentes roles: evaluador, ejecutor, observador, anotador y el árbitro. Por ejemplo: en un ejercicio de lanzar superando el brazo, habría el ejecutor, el observador (miraría qué recursos utiliza el lanzador para superar el brazo), el anotador (apuntaría cuántos goles ha metido y por dónde los ha metido), un árbitro pitaría falta o no, miraría si está fuera de los $5 \mathrm{~m}$, etc.).

La micro-enseñanza: se ve reflejada, por ejemplo, cuando el portero cadete transmite al portero infantil los desplazamientos laterales propios de la posición de portero.

\subsubsection{EE que propician la socialización}

Aquí el juego de roles, la simulación social y el trabajo en dinámicas de grupo se basan en los objetivos sociales y en los contenidos de actitudes, normas y valores.

En waterpolo no solo lo podemos utilizar dentro del agua, sino también fuera. Sobre todo en las categorías inferiores, para que entiendan y se pongan en la piel de otras personas, y reflexionen sobre ciertas actitudes. Por ejemplo, simulamos un partido con padres y madres, jugadores o jugadoras, árbitro y entrenador o entrenadora, entre otros. Cada uno tiene su rol. Al final ponemos en común lo que hemos visto y qué cosas se podrían mejorar. También se podría hacer añadiendo un comportamiento, generalmente incorrecto, que potencia el entrenador real. De esta manera, observaríamos cómo se resolvería el conflicto, que finalizaría con una puesta en común en la que se proponen correcciones y mejoras.

\subsubsection{EE que implican cognoscitivamente de manera más directa al alumnado}

El descubrimiento guiado se daría, por ejemplo, en un equipo infantil en el que defienden haciendo pressing. Si hay falta ayudarán al boya (si el defensor está por detrás), ayudarán al lateral (si el defensor de boya está por delante), harán brazo (si tiene buena posición para lanzar, si se acaba el tiempo de posesión, si es muy buen lanzador $u$ otra circunstancia...). El defensor elige la manera que cree correcta y el entrenador le va guiando hasta conducirlo a la respuesta deseada, que buscaremos mediante el refuerzo de respuestas correctas y el rechazo de las erróneas (Ver Figura 5).

La resolución de problemas se utilizaría, por ejemplo, cuando le pedimos al jugador o jugadora que flote por el lado débil (donde no está la pelota). Primero, el jugador o jugadora comentaría la manera que considera correcta. Luego dejaríamos que el jugador o jugadora actuara libremente ante el ataque e iríamos diciendo cuáles son válidas. Por ejemplo, probando hacerlo de manera vertical o de manera horizontal.

\subsubsection{EE que favorecen la creatividad}

Las posibilidades de este estilo son innombrables. Por ejemplo: un jugador o jugadora inventa una tarea para trabajar el pase, y los otros lo hacen.

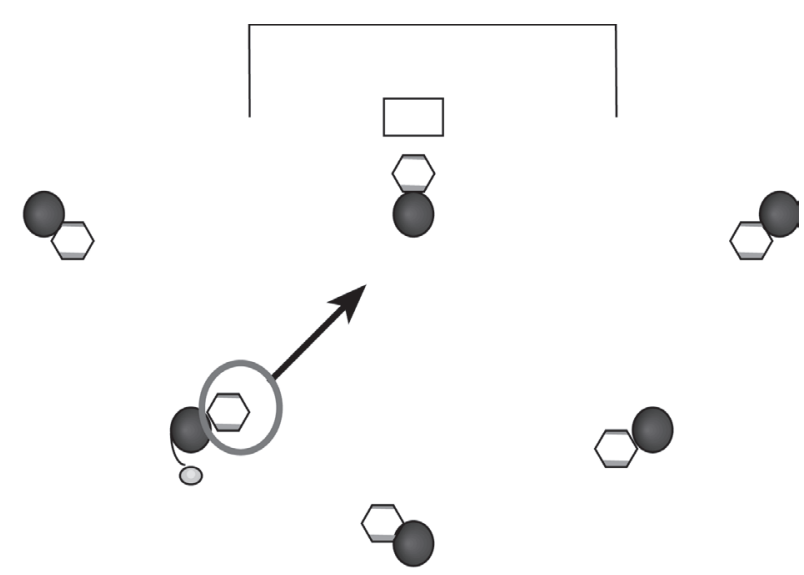

Figura 5. Falta y ayuda (descubrimiento guiado). El jugador que defiende la posición 4, señalado con el círculo rojo, realiza falta y debe escoger una de las cuatro opciones que tiene para ayudar a su equipo. Como entrenadores deberemos dejar que el defensor tome la decisión, y si no elige la correcta (en este caso, imaginándonos que quedan 18 s de posesión todavía, sería ayudar al boya, ya que el defensor está por detrás), tendremos que ir guiándolo hacia ella.

Por ejemplo: si elige hacer la ayuda lateral a posición 3, le preguntaremos: ¿el defensor de 3 está en línea de pase o no? La respuesta será que sí, y él mismo se dará cuenta de que ahí no debía efectuarse la ayuda. Le preguntaremos: ¿a quién crees que debes ayudar?, y, si no lo sabe, añadiremos: ¿por dónde está defendiendo el defensor de boya?, y, así, gradualmente. 


\subsection{Técnica de enseñanza}

Según Delgado (1993), la técnica de enseñanza (TE) se define como la forma en que el entrenador transmite la información y las habilidades que quiere enseñar. Este procedimiento se utiliza para dirigir las actividades de los jugadores hacia un objetivo de aprendizaje. Por un lado se debe tener en cuenta la presentación de las tareas y actividades, y por otro lado las reacciones del entrenador ante la actuación y ejecución de los jugadores. Es decir, por un lado la información inicial, y por otro lado la información de retorno. Delgado (1991), Delgado (1993) y Sánchez Bañuelos (1990) coindicen en destacar dos tipos de técnicas de enseñanza: mediante la instrucción directa y mediante indagación.

\subsubsection{TE mediante la instrucción directa}

Aporta un modelo correcto de ejecución a través de la información inicial de tipo visual, verbal o mixta. Los ajustes posteriores se llevan a cabo por el conocimiento de la ejecución y de los resultados (feedback). Se entiende que existe una solución que da cierto rendimiento y está bien definida (modelo), que surge de forma empírica y que precisa de estudios biomecánicos para su optimización. Esta técnica trata la ejecución de los modelos, pero no los problemas contextuales de cuándo y cómo.

\subsubsection{TE mediante la indagación}

El profesor o el entrenador tratan de inducir la actividad que han de realizar los jugadores indicando las normas para que la desempeñen. Tiene que presentar la situación que hay que explorar o el problema motor adecuadamente, y dar información adicional en función de las respuestas. Las correcciones son de tipo general. Los juegos o formas jugadas para plantear problemas situacionales favorecen la motivación por la búsqueda.

\subsection{Estrategia de práctica}

La estrategia de práctica (EP) es la forma particular de abordar los diferentes ejercicios que componen la progresión de enseñanza de una determinada habilidad motriz. Dependiendo de la complejidad de esta habilidad motriz y de la manera que tiene el entrenador de presentar y dirigir los contenidos a los jugadores, utilizaremos un tipo de estrategia u otra (Sicilia \& Delgado, 2002).

Delgado (1993) afirma que existe una gran variedad de estrategias y de combinaciones posibles, y presenta estrategias analíticas (análisis puro, secuencial y progresivo) y estrategias globales (global con modificación de la situación real, global con polarización de la atención y global puro).

En el caso del waterpolo, utilizar una estrategia de práctica de carácter analítico puro sería el caso del jugador solo que realiza, por ejemplo, movimientos de brazo. Debemos proporcionarle los ítems para que lo ejecute correctamente: el brazo por delante, la mano abierta y tensa, el brazo tenso, las rodillas altas, etc.
A partir de aquí aparecería la escala de «grises», a la que de manera progresiva iríamos añadiendo más jugadores. Por ejemplo: tres jugadores que atacan (se pasan la pelota entre ellos y obligatoriamente tienen que aguantarla 3s, para que al defensor le dé tiempo a realizar bien el movimiento de poner el brazo, y así lo vaya automatizando). El jugador que pone el brazo lo hará de una manera u otra en función de por dónde le venga el pase de su atacante. De esta manera, cada vez añadiríamos más jugadores, tanto atacantes como defensores, hasta llegar al juego real, carácter global puro, en el que el jugador decida cómo y cuándo poner el brazo.

\section{Propuesta pedagógica para el waterpolo}

Para jugar a waterpolo precisa la reproducción de los modelos técnicos y la resolución de situaciones individuales y colectivas, ya que es un deporte de colaboración/oposición en un espacio común. La técnica y la táctica están directamente relacionadas, por eso su enseñanza se realiza de forma simultánea. Nos ayudaremos del juego reducido, pues facilitaremos el aprendizaje al ser más simple.

Mediante las diferentes tareas que propongamos podremos trabajar los contenidos y conseguir los objetivos marcados. Estas tareas deben ser variadas y enriquecedoras, de manera que permitan una participación activa y que sean adecuadas, siempre teniendo en cuenta qué jugadores y jugadoras tenemos y cuáles son sus requerimientos.

Según Sampedro (1999), en las situaciones que proponemos, el juego y las posibilidades de los que aprenden tienen que estar equilibradas. Estos serían los pasos que seguir, de menor a mayor exigencia: juegos, «mini-waterpolo», perfeccionamiento del waterpolo, waterpolo.

Siguiendo esta afirmación, nos encontraremos con casos en los que el proceso de enseñanza-aprendizaje no se podrá hacer de manera tan gradual, ya que no todos los jugadores empiezan en la misma etapa o franja de edad. Según el nivel del grupo, el espacio, el material, el técnico, los recursos humanos, económicos, y otros condicionantes, seguiremos las etapas con mayor o menor rigurosidad. Cada etapa vendrá muy marcada por las anteriores. Por ejemplo: no es lo mismo trabajar con una selección infantil, en la que se encuentran los mejores de su categoría y en la que muchos jugarán también con categorías superiores, y, por lo tanto, podremos desarrollar las habilidades técnicas, tácticas y estratégicas de cadetes que trabajar con un equipo infantil, cuyos jugadores acaban de empezar a jugar, porque primero tendríamos que desarrollar las habilidades básicas propias de las categorías benjamines y alevines.

Autores como Delgado (1985) y Hernández (1987) ponen atención al revisar la intervención didáctica en la enseñanza de los juegos deportivos, la cual está excesivamente centrada en los modelos estereotipados de ejecución técnica, para centrarse más en la estruc- 
tura interna de los juegos deportivos y en el desarrollo de la conducta de decisión. Medina (1997) apuesta por una técnica de enseñanza mixta a lo largo del tiempo, empezando, en primer lugar, por la indagación, en segundo lugar por la instrucción directa, para volver otra vez a la indagación; mientras que Conde y Pérez (2003) realizan una propuesta de los estilos de enseñanza en natación que va desde lo analítico a lo global.

En la misma línea, Moreno (1995) y Devís (1990) apuestan por una enseñanza de la técnica basada en juegos modificados, para pasar después al aprendizaje técnico. Estos autores enfatizan tres aspectos que pueden posibilitar un cambio en la enseñanza, en el que el jugador o jugadora comprenda, en su significado más profundo, lo que está haciendo (la toma de decisiones y la formulación de juicios, la comprensión de los contextos de los juegos deportivos y la importancia de la táctica en la iniciación deportiva).

Riera (1995) afirma que es necesario conocer la esencia del juego y sus principios tácticos, que a su vez vendrán dados por indicadores de la táctica deportiva, para poder sacar un mayor partido de la técnica. Por otro lado, Blázquez (1986) señala que la contribución del entrenador consiste en crear una situación problemática y concienciar de ella a los jugadores, conducirlos a las respuestas a partir de sus propios conocimientos y analizar la solución descubierta y utilizada.

No hay un estilo de enseñanza perfecto. Hay diferentes maneras de enseñar en función de las tareas y de los objetivos que nos propongamos. Los entrenadores tendremos que decidir qué estilo o estilos utilizar, en qué momento y con qué grupo o categoría.

Siguiendo la clasificación de Delgado (1993) y profundizando en lo expuesto anteriormente, las etapas son las siguientes: etapa de iniciación, etapa de desarrollo, etapa de perfeccionamiento y etapa de rendimiento.

\section{ETAPA DE INICIACIÓN}

En esta etapa nos basaríamos en el juego cuyo objetivo es adquirir el gusto por el propio juego y su participación en este. Los juegos han de tener en cuenta la distribución del tiempo y del espacio, y, sobre todo, el carácter lúdico. En esta etapa deberían acabar dominando el medio, el juego individual y el espacio táctico. Fomentaremos la capacidad coordinativa, la perceptiva/decisional, la de socialización y la de respeto por las normas.

Con los juegos genéricos se pretende desarrollar unas habilidades básicas sobre las que se van añadiendo, de manera progresiva, otras técnicas y tácticas. Hay juegos que estarán más dirigidos a la estructura interna del waterpolo (los diez pases, el waterpolo-torre), y otros más enfocados a contenidos técnicos (rondós, juegos de lanzamiento). En esta etapa predominarán los juegos o las formas jugadas, pero también incluiremos ejercicios y partidos en los que el entrenador podrá modifi- car las reglas, el espacio o el material según el objetivo que persiga.

En este estadio entrarían las categorías pre-benjamín, benjamín y alevín, que disponen de una normativa adaptada para estas edades.

Teniendo en cuenta los EE de Delgado (1993), trabajaríamos mezclando los EE que posibilitan la participación de los jugadores en la enseñanza, que fomentan la individualización (no programas individuales), que propician la socialización, que son cognoscitivos y que favorecen la creatividad. Preferentemente, utilizaremos la TE mediante la indagación, aunque podremos introducir pinceladas de la instrucción directa. La EP será, mayoritariamente, de carácter global.

De manera gradual, utilizaremos los estilos, las técnicas de enseñanza y la estrategia de la práctica, es decir, en la categoría pre-benjamín emplearemos mucho los estilos cognoscitivos que favorecen la creatividad, las técnicas mediante la indagación y la estrategia global pura. A medida que pasen a la categoría benjamín y alevín, aplicaremos la escala de «grises» hasta llegar a la siguiente etapa o al final de la categoría alevín, en la que, progresivamente, iremos otorgándole mayor importancia a la instrucción directa, a los estilos de enseñanza tradicionales y a la estrategia de carácter más analítico.

\section{ETAPA DE DESARROLLO}

En esta etapa entraría en juego el «mini-waterpolo», que también tiene unas normas adaptadas a las necesidades en el caso infantil. Englobaría las categorías infantil y cadete.

Esta etapa vendrá muy marcada por la anterior. Los contenidos técnicos-tácticos irán apareciendo de forma coherente a lo largo de la etapa para evitar errores muy frecuentes cuando se precipitan los acontecimientos deportivos y no se respetan temporalmente los grandes criterios de optimización del individuo. Tendríamos que fomentar las capacidades condicionales, seguir trabajando las habilidades básicas, desarrollar las capacidades de competencia socio-motriz básicas de ataque y defensa, y desarrollar las capacidades de competición y superación personal y colectiva.

Utilizando la clasificación de Delgado (1993), trabajaríamos mezclando todos los EE, pero, sobre todo, recalcaríamos los estilos tradicionales en la categoría infantil, ya que es una edad ideal para trabajar las principales habilidades técnicas y progresar adecuadamente. Una vez pasen a cadete, si los contenidos se han asimilado correctamente, seguiremos trabajando con estilos tradicionales, pero le concederemos mucha más importancia a los estilos que posibilitan la participación del jugador en la enseñanza que los que fomentan la individualización (no programas individuales), los que propician la socialización, los cognoscitivos y los que favorecen la creatividad.

Aunque muchos deportes colectivos están en contra de la TE mediante instrucción directa, creemos que en nuestro deporte, por sus características, al no practicar- 
se en nuestro medio natural, y porque si no se trabajan las principales habilidades básicas y técnicas de manera correcta tendríamos mucho desgaste energético, utilizaremos, sobre todo en categoría infantil, la instrucción directa. No obstante, nunca dejaríamos a un lado el trabajo mediante la indagación. Cuando pasen a cadete, si han asimilado correctamente los contenidos, volveremos a conceder mayor importancia al resto de estilos de enseñanza.

La EP será mayoritariamente de carácter analítico. También utilizaremos la estrategia de la práctica global, pero con menor frecuencia. Cuando pasen a cadete, si han asimilado correctamente los contenidos, será a la inversa.

\section{ETAPA DE PERFECCIONAMIENTO}

Para el perfeccionamiento del waterpolo los principales objetivos serían los siguientes: desarrollar la fuerza, como cualidad física básica para llegar a jugar en la alta competición (sin olvidarnos de las otras capacidades condicionales); dominar el juego individual; dominar las intenciones tácticas individuales; entrenar y dominar el juego colectivo; así como entrenar y dominar los sistemas de juego.

En esta etapa entrarían las categorías juvenil y equipos B. Una vez conseguidas las habilidades básicas y las principales habilidades técnicas y tácticas, volveríamos a trabajar como en el primer estadio.

Tomando como referencia los EE de Delgado (1993), mezclaríamos los estilos que posibilitan la participación del jugador en la enseñanza, que fomentan la individualización, que propician la socialización, cognoscitivos y que favorecen la creatividad. Preferentemente, utilizaremos la TE mediante la indagación, aunque introduciremos pinceladas de instrucción directa. La EP será, mayoritariamente, global. También emplearemos el análisis puro, secuencial y progresivo, pero en menor medida, $y$, sobre todo, como calentamiento específico de la parte principal de la sesión.

\section{ETAPA DE RENDIMIENTO}

En el waterpolo de rendimiento trabajaríamos de manera muy similar a la etapa anterior. Dependiendo de las características del grupo, haríamos más hincapié en unos estilos y técnicas u otros. Entraría la categoría absoluta. Esta combinación de estilos, técnicas y estrategias es lo que favorecerá la adquisición y riqueza del aprendizaje de los jugadores. (Ver Tabla 1).

La manera de evaluar el aprendizaje sería midiendo $\mathrm{y}$, posteriormente, interpretando aquello que se quiere entrenar. Los instrumentos para evaluar nos proporcionarán los indicadores que nos permitirán obtener información de aquello enseñado. La evaluación de waterpolo es muy difícil de efectuar, ya que hay múltiples variables, por lo tanto, tendremos que concretar mucho nuestros instrumentos y determinar qué criterios utilizaremos para que la evaluación sea útil.

\section{Conclusiones y líneas de futuro}

Si nos fijamos en la metodología del waterpolo referenciada, nos daremos cuenta de que existe muy poca información al respecto. En este punto está la aportación del artículo: descifrar los estilos de enseñanza aplicados al waterpolo y cubrir la falta de información detectada en este ámbito.

Consideramos que las tareas propuestas serán acertadas si existe correspondencia entre la dificultad de éstas y el nivel de capacidad de los jugadores y las jugadoras. Para que el proceso de formación deportiva sea el correcto deberemos tener como base el aprendizaje de los contenidos básicos del juego.

Nuestra propuesta se basa en una enseñanza mixta. En ningún momento se excluye una técnica de enseñanza, una estrategia de práctica o un estilo de enseñanza específica. Se trata de utilizarlos todos, pero concediendo mayor importancia a unos $u$ otros en función de la categoría o el nivel en el que se encuentre el grupo. Así, dependiendo de la categoría a que pertenezca o al nivel adquirido, aunque actuando de

Tabla 1. Propuesta didáctica de las diferentes etapas del waterpolo (WP).

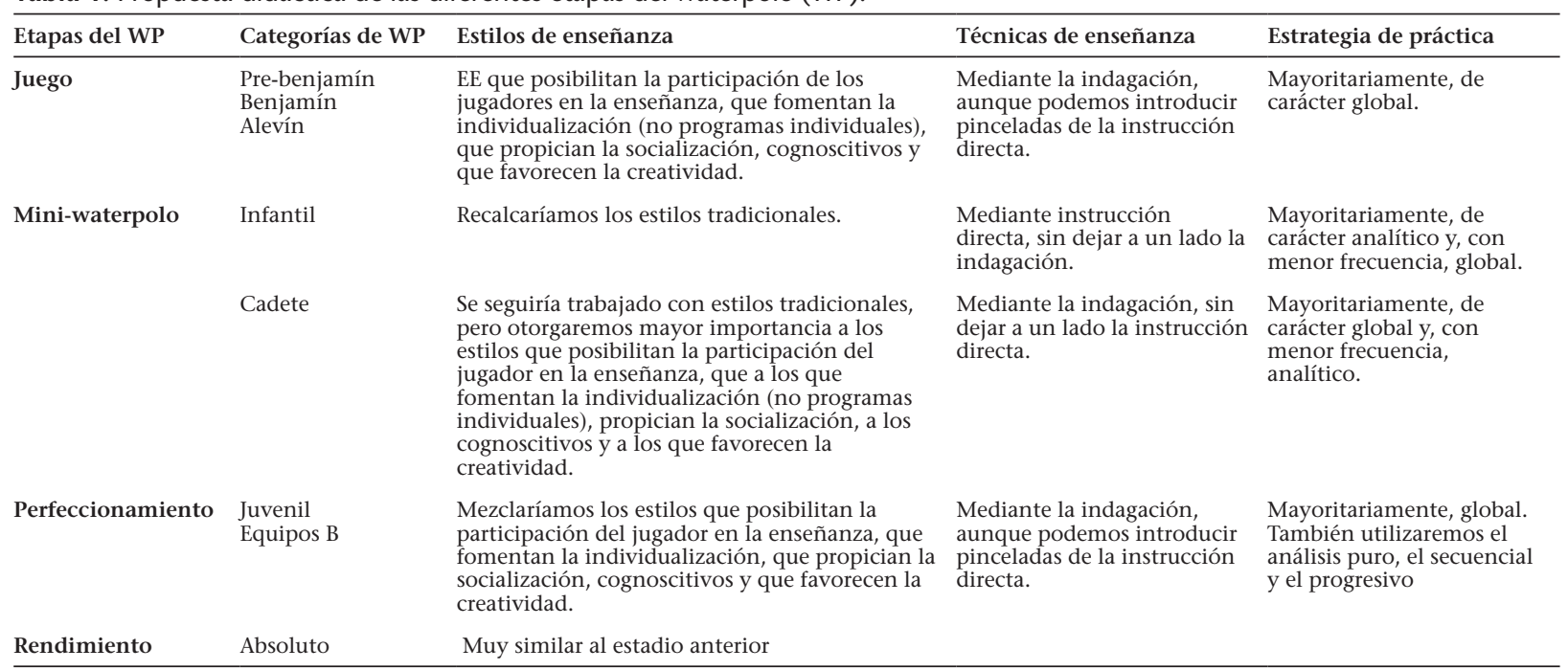


manera simultánea, predominará la técnica de enseñanza, la estrategia de práctica o el estilo en mayor o menor grado.

Como líneas de futuro creemos que hay que plantearse la aplicación directa de la propuesta y poder evaluar la metodología utilizada. Deberemos tener en cuenta que los objetivos y/o contenidos perseguidos sean suficientes en cuanto al número y el nivel que se desea conseguir, y que el desarrollo del programa sea adecuado para las edades y acorde a la planificación real.

\section{Referencias}

Argudo, F. (1996). Niveles de enseñanza en el aprendizaje del waterpolo. Agua y Gestión - SEAE, 35, 19-27.

Argudo, F. \& García, A. (1996). Enseñanza de la táctica en el waterpolo (I). Comunicaciones técnicas, 1, 21-29.

Blázquez, D. (1986). Iniciación a los deportes de equipo. Barcelona: Martínez Roca.

Blázquez, D. (1995). La iniciación deportiva y el deporte escolar. Barcelona: INDE.

Cancela, J. M., \& Lago, C. (2001). El proceso de iniciación deportiva en el waterpolo. Secuenciación de los contenidos técnico-tácticos. Comunicaciones técnicas, 1, 9-17.

Conde, E. \& Pérez, A. (2003). Los estilos de enseñanza en la natación. De lo analítico a lo global $\left(1^{\mathrm{a}}\right.$ parte). Comunicaciones técnicas, 6, 43-52.

Delgado, M.A. (1985). El planteamiento por problemas: una alternativa a la enseñanza y entrenamiento del fútbol. En VV.AA. (1985) Criterios para la mejora de nuestro fútbol. Madrid: Gymnos.

Delgado, M.A. (1991a). Hacia una clarificación conceptual de los términos en didáctica de la Educación Física y el Deporte. Revista de Educación Física. Renovar teoría y práctica, 40, 2-10.

Delgado, M.A. (1991b). Estilos de Enseñanza en Educación Física. Propuesta para una Reforma de la Enseñanza. Granada: I.C.E. de la Universidad de Granada.

Delgado, M.A. (1993). Los métodos didácticos en Educación Física. En VV.AA. Fundamentos de Educación Física para enseñanza primaria. Vol II. Barcelona: INDE.

Devís, J. (1990). Renovación pedagógica en la educación física: hacia dos alternativas de acción II. Perspectivas de la actividad física y del deporte, 5, 13-16.

Fernández, A. (2004). Unidad didáctica: aprende a jugar a waterpolo. Comunicaciones técnicas, 2, 25-36.

Feu, S. (1999). Iniciación al waterpolo: Mejora perceptiva del jugador con balón. Comunicaciones técnicas, 3, 27-33.

García, M. (1999). Centro de waterpolo. Creación de un grupo de edades. Comunicaciones Técnicas, 2, 29-44.

García, M. (2000). Iniciación al waterpolo. Madrid: Editorial Gymnos.

Genoves, R. (1992). Ejemplo de trabajo para waterpolo de base. Comunicaciones técnicas, 4, 43-63.
Herbert, J. (1967). A system of Analysing Lessons. New York: Teachers College Press.

Hernández, J. (1987). Estudio sobre el análisis de la acción del juego en los deportes de equipo: aplicación al baloncesto. (Tesis doctoral no publicada). Universidad de Barcelona.

López, J. (1995). Una propuesta para la estructuración de niveles en la iniciación al waterpolo. Comunicaciones técnicas, 1, 13-16.

Lloret, M. (1994). Análisis de la acción de juego en el waterpolo durante la Olimpiada de 1992. (Tesis Doctoral no publicada). Universidad de Barcelona, Barcelona.

Lloret, M., \& Baella, O. (1996). El waterpolo en la escuela: Una propuesta programática. Comunicaciones técnicas, 5, 19-26.

Medina, J. (1997). Técnicas de Grupo como medio de formación del profesorado de Educación Física. Aplicaciones en la práctica. Revista Española de Educación Física y Deportes, 4(1), 16-23.

Moreno, J. A. (1995). Descubrimiento del waterpolo a través del juego. Comunicaciones técnicas, 6, 43-50.

Mosston, M. \& Ashworth, S. (1986). Teaching physical education. New York: Macmillan Publishing Company.

Mosston, M. \& Ashworth, S. (1993). La enseñanza de la Educación Física. La reforma de los Estilos de Enseñanza. Barcelona: Hispano Europea.

Riera, J. (1995). Análisis de la táctica deportiva. Apunts: Educacion Física y Deportes, 40, 47-60.

Ortega, J.L. (1996). Una propuesta para la iniciación al waterpolo. Comunicaciones técnicas, 2, 27-34.

Parlebas, P. (1981). Contribution à un lexique commenté en science de l'action motrice. París: INSEP

Sampedro, J. (1999). Textos Federación Española de Baloncesto. Cursos de Entrenador de Baloncesto. Iniciación y Primer nivel. Málaga: Instituto Andaluz del Deporte.

Sánchez Bañuelos, F. (1990). Bases para una didáctica de la Educación Física y el Deporte. (2ª Edición ampliada). Madrid: Gymnos.

Sicilia, A. (2001). La investigación de los estilos de enseñanza en la educación física. Un viejo tema para un nuevo siglo. Sevilla: Wanceulen.

Sicilia, A. \& Delgado (2002). Educación Física y Estilos de Enseñanza. Barcelona: INDE.

Stones, E. (1973). Voyage of discovery, Education, 3-15(1), 18-29.

Vila, H., Ferragut, C., Abraldes, J.A., Rodríguez, N. \& Argudo, F.M. (2010). Caracterización antropométrica en jugadores de elite de waterpolo. Revista Internacional de Medicina y Ciencias de la Actividad Física $y$ el Deporte, 10(40), 652-663.

\section{Proposta didàctica en l'ensenyament} del waterpolo

Resum. El waterpolo requereix una sèrie de fonaments tècnics, tàctics, de qualitats psicosocials i de preparació física que els jugadors $i$ les jugadores aniran assolint de manera progressiva amb l'edat. 
102 | Noma 2016, 34(1)

La metodologia d'aprenentatge és molt important a l'hora d'ensenyar qualsevol esport. A més, el waterpolo es practica en el medi aquàtic, que no és el medi natural de l'ésser humà, la qual cosa li dóna una especial singularitat respecte la resta d'esports col-lectius. A la nostra disciplina, les referències bibliogràfiques relatives a com dur a terme aquest procés són limitades i creiem que s'ha de plantejar, a més de què ensenyar, com i quan ensenyar-lo.
Yurema Sabio-Lago, Josep Solà-Santesmases \& Myriam Guerra-Balic

El nostre objectiu és fer una proposta dels plantejaments pedagògics que cal seguir en les diferents etapes d'ensenyament del waterpolo tenint en compte els objectius $i$ continguts que s'hauran d'assolir en cadascuna de les categories.

Paraules clau: esport; waterpolo; plantejament pedagògic; estils d'ensenyament 